\title{
A NECESSARY AND SUFFICIENT CONDITION THAT A CURVE LIE ON A HYPERQUADRIC
}

\author{
LOUIS C. GRAUE
}

Introduction. The purpose of this paper is to give a necessary and sufficient condition in terms of the ordinary curvatures of a curve for it to lie on any real central hyperquadric in a Euclidean space $\Re_{n}$. This problem was solved for the hypersphere in $\Re_{n}$ by Karel Havliček in his paper Contact des courbes et des hypersphères dans un espace euclidean à n dimensions.-Courbes sphériques, which appears in Casopis pro Pěstování Matematiky a Fysiky vol. 72 (1947).

First we find a necessary and sufficient condition in terms of socalled hyperquadric curvatures for a curve to lie on a given real central hyperquadric. Then by means of invariant functions in $\$ 3$ we find the solution of our problem.

1. Fundamental concepts. We shall begin by finding a necessary and sufficient condition that a curve lie on a hyperquadric homothetic to a given hyperquadric, where by a hyperquadric we always mean a real central hyperquadric whose equation may be written in the form

$$
\sum_{1}^{n} a_{b c}\left(x^{b}-c^{b}\right)\left(x^{c}-c^{c}\right)-\gamma_{0} d^{2}=0
$$

with given real coefficients $a_{b c}=a_{c b}$ and center $p\left(c^{1}, \cdots, c^{n}\right)$, where the determinant $\left[a_{b c}\right]=1, \gamma_{0}= \pm 1$, and $d$ is a real number. If $d=0$, then (1.1) is a hypercone with vertex $p$. If $d \neq 0$, then the number $|d|$ is called the radius of the hyperquadric (1.1). The coefficients $a_{b c}$ are regarded here as the components of a metric tensor.

We shall use the notation $\bar{r}$ to denote the radius vector of a real curve $\bar{r}=\bar{r}(t)$, where by a curve we shall always mean a general curve defined in the following definition.

Definition 1.1. A real curve $\mathfrak{S}$ of $\Re_{n}$ is called a general curve if it has the following properties:

(a) it is nonisotropic with respect to $a_{b c}$;

(b) it has $n$ different osculating spaces;

(c) its $k$ th osculating space is not tangent to the isotropic hypercone of $a_{b c}(k=1, \cdots, n-1)$.

By means of the metric $a_{b c}$ we introduce all the usual notions of a tensor calculus. Hence for instance $\bar{u} \cdot \bar{v} \equiv a_{b c} u^{b} v^{c}$ is a scalar product of two vectors, and so on. In particular $\bar{u} \cdot \bar{v}=0$ means that the vectors

Received by the editors August 14, 1950. 
$\bar{u}$ and $\bar{v}$ are conjugate (with respect to our hyperquadric). The hyperquadric arc $\mathfrak{S}$ of $\mathbb{S}$ is defined by $d \mathfrak{S}=(|d \bar{r} \cdot d \bar{r}|)^{1 / 2}$ where $\bar{r}=\bar{r}(t)$ are the equations of $\mathfrak{E}$. Henceforth we put $\bar{r}(t)=\bar{\Re}(\mathfrak{S})$ and denote derivatives with respect to $\mathfrak{S}$ by accents (that is, ${\overline{\mathfrak{R}^{\prime}}}^{\prime}=d \bar{\Re} / d \mathfrak{S}$, and so on).

The Frenet formulas for $\left(\mathbb{S}\right.$ (with respect to $a_{b c}$ ) are

$$
\overline{\mathfrak{N}}_{i}^{\prime}=-\gamma_{i} \gamma_{i+1} \Omega_{i} \bar{\Re}_{i-1}+\Omega_{i+1} \bar{\Re}_{i+1} \quad\left(\Omega_{0}=\Omega_{n}=0\right)
$$

where $\overline{\mathfrak{N}}_{i}(i=0,1, \cdots, n-1)$ are mutually hyperquadric conjugate unit vectors with $\overline{\mathfrak{N}}_{i} \cdot \overline{\mathfrak{N}}_{i}=\gamma_{i+1}\left(\gamma_{i+1}= \pm 1\right)$ and $K_{i}>0(i=1, \cdots$, $n-1)$ are the hyperquadric curvatures.

It may be shown that the $K_{i}$ define a curve up to initial conditions. We shall designate by $\Re_{i}$ the radius of the hyperquadric curvature $K_{i}$ (that is, $\Re_{i}=1 / \Re_{i}$ ). The hyperplane spanned by the vectors $\overline{\mathfrak{N}}_{i}(i=1, \cdots, n-1)$ at the point $\bar{\Re}(\Im)$ of the curve $\bar{\Re}=\bar{\Re}(\Im)$ will be termed the conjugate hyperplane of the curve.

2. Contact of a hyperquadric and a curve. Let $\bar{x}$ be the radius vector of a variable point in $\Re_{n}$. Let $\bar{p}$ be the radius vector of any fixed point and $r$ any real numerical constant. Then any hyperquadric homothetic to (1.1) and having center $\bar{p}=\left(x_{0}^{1}, \cdots, x_{0}^{n}\right)$ and radius $r$ has one of the equations

$$
(\bar{x}-\bar{p}) \cdot(\bar{x}-\bar{p})-\gamma r^{2}=0, \quad \gamma= \pm 1 .
$$

If $r=0$ then this equation represents a hypercone. We shall never take the point $\bar{\Re}_{0}$ of the curve $\bar{\Re}=\bar{\Re}(\Im)$ at the vertex of this hypercone.

DEFINITION 2.1. The power $\mathfrak{B}$ of an arbitrary point $\bar{\Re}$ in relation to the hyperquadric $(2.1)$ is defined by the expression $\mathfrak{B}=(\bar{\Re}-\not{\not})$ $\cdot(\bar{\Re}-\not)-\gamma r^{2}$.

Definition 2.2. Consider a hyperquadric (2.1) which goes through the point $\bar{\Re}_{0}$ of the curve $\bar{\Re}=\bar{\Re}(\Im)$ and denote by $\mathfrak{B}(\widetilde{S}, 0)$ the power of the point $\bar{\Re}(\Im)$ of the curve with respect to the hyperquadric (2.1). Then if $\lim \mathfrak{S}_{=0}\left(\mathfrak{B}(\mathfrak{S}, 0) / \mathfrak{S}^{p}\right)=0, p=1, \cdots, q$, we say that the hyperquadric (2.1). has at the point $\bar{\Re}_{0}$ a contact with the curve $\bar{\Re}=\bar{\Re}(\mathfrak{S})$ of at least order $q$.

We may prove the following theorem.

THEOREM 2.1. A necessary and sufficient condition that a hyperquadric (2.1) through the point $\bar{\Re}_{0}$ of a curve $\bar{\Re}=\bar{\Re}(\Im)$ have a contact of at least order $q=1$ with the curve at this point is that its center lie in the conjugate hyperplane of the curve at the point $\bar{\Re}_{0}$.

We shall discuss the significance of contact of order $q>1$ later in 
Theorem 2.5. We may easily prove the next result.

TheOREM 2.2. A necessary and sufficient condition that a curve lie on a hyperquadric (2.1) is that all its conjugate hyperplanes have at least one fixed common point and that this point is the center of the hyperquadric on which it lies.

Definition 2.3. Consider a curve $\bar{\Re}=\bar{\Re}(\Im)$ and any fixed point $\bar{p}$. Then we may write

$$
\bar{\Re}-\bar{P}=\sum_{i=0}^{n-1} \mathfrak{E}_{i} \bar{\Re}_{i} .
$$

The functions $\mathfrak{S}_{i}(i=0,1, \cdots, n-1)$ will be called the relative curvatures of the curve $\bar{\Re}=\bar{\Re}(\Im)$ with respect to the point $\bar{p}$.

From (2.2) we have $\mathfrak{夭}_{i}=\gamma_{i+1}(\bar{\Re}-\bar{p}) \cdot \overline{\mathfrak{\Re}}_{i}$ and by differentiation we obtain the equations:

$$
\begin{aligned}
& \mathfrak{G}_{0}^{\prime}=\gamma_{1} \gamma_{2} \Re_{1} \mathfrak{G}_{1}+1, \\
& \mathfrak{S}_{i}^{\prime}=-\Re_{i} \mathfrak{S}_{i-1}+\gamma_{i+1} \gamma_{i+2} \mathfrak{\Omega}_{i+1} \mathfrak{G}_{i+1} \quad(i=1, \cdots, n-2), \\
& \mathfrak{C}_{n-1}^{\prime}=-\Re_{n-1} \mathfrak{C}_{n-2} \text {. }
\end{aligned}
$$

From these we may show that the relative curvatures determine a curve up to initial conditions. From the equation $(\bar{\Re}-\bar{P}) \cdot(\bar{\Re}-\bar{P})$ $=\sum_{i=0}^{n-1} \gamma_{i+1} \mathfrak{E}_{i}^{2}$ it is evident that the relative curvatures also determine the hyperquadric length of the vector $\bar{\Re}-\bar{p}$.

TheOREM 2.3. A necessary and sufficient condition that a curve $\bar{\Re}=\bar{\Re}(\Im)$ lie on the hyperquadric (2.1) is that there exists a point $\bar{p}$ such that

$$
\sum_{i=1}^{n-1} \gamma_{i+1} \mathfrak{S}_{i} \mathfrak{S}_{i}^{\prime}=0 \quad\left(\mathfrak{G}_{0}=0\right)
$$

at each of the points of the curve, where the $\mathfrak{S}^{\prime}$ 's are given by the recursion formulas

$$
\begin{aligned}
\mathfrak{S}_{i}=\gamma_{i} \gamma_{i+1} \Re_{i} \mathfrak{S}_{i-1}^{\prime} & +\gamma_{i} \gamma_{i+1} \Re_{i} \Re_{i-1} \mathfrak{S}_{i-2} \\
& (i=1, \cdots, n-1), \Re_{0} \mathfrak{C}_{-1}=-1 .
\end{aligned}
$$

The proof is based on the fact that $\sum_{i=0}^{n-1} \gamma_{i+1} \Subset_{i}^{2}=$ const. is a necessary and sufficient condition for the curve to lie on (2.1) and on using equation(2.3). 
For $n=3$ the condition (2.4) becomes $\gamma_{2} \Re_{1}+\gamma_{3}\left(\Re_{1}^{\prime} \Re_{2}\right)^{\prime} \Re_{2}=0$. If our given quadric is a sphere, then $\gamma_{1}=\gamma_{2}=\gamma_{3}=1$ and the $\Re$ 's are the ordinary radii of curvatures. This condition then becomes the condition for a curve to be spherical.

TheOREM 2.4. A necessary and sufficient condition that a curve $\bar{\Re}=\bar{\Re}(\Im)$ lie on a hypercone (equation (2.1) with $r=0$ ) is that at least one of the equations

$$
\begin{aligned}
&-\gamma_{n} \sum_{i=1}^{n-2} \gamma_{i+1} \mathfrak{\Xi}_{i}\left(-\Re_{i} \mathfrak{\Xi}_{i-1}+\gamma_{i+1} \gamma_{i+2} \Re_{i+1} \mathfrak{E}_{i+1}\right) \\
& \pm \Re_{n-1} \mathfrak{S}_{n-2}\left(-\gamma_{n} \sum_{i=1}^{n-2} \gamma_{i+1} \mathfrak{\Xi}_{i}^{2}\right)^{1 / 2}=0
\end{aligned}
$$

is satisfied at each of the points of the curve.

This is a special case of Theorem 2.3 in which we make use of the equation $\sum_{i=1}^{n-1} \gamma_{i+1} \S_{i}^{2}=0$.

TheOREM 2.5. Let the hyperquadric (2.1) have a contact of at least order $q(2 \leqq q \leqq n)$ with the curve $\bar{\Re}=\bar{\Re}(\Im)$ at the point $\bar{\Re}_{0}$. Then the relative curvatures $\mathfrak{S}_{1}, \cdots, \mathfrak{S}_{q-1}$ are uniquely determined while the relative curvatures $\mathfrak{E}_{q}, \cdots, \mathfrak{S}_{n-1}$ are arbitrary.

This theorem may be proved by induction on $q$ and by using the formulas (1.2) in the equations for the derivatives of the power $\mathfrak{P}(\mathfrak{S})$.

3. The invariant parameter. We shall now derive a necessary and sufficient condition in terms of the ordinary curvatures of a curve that it lie on any real central hyperquadric.

We shall write $\bar{r}=\bar{r}(s)$ when the parameter used to describe the curve is the ordinary arc length $s$. All derivatives with respect to this parameter will be denoted by dots (that is, $d \bar{r} / d s=\bar{r}$, and so forth). The ordinary tangent, normals, curvatures, radii of curvature, and so forth, will be denoted by small letters $\left(\bar{t}, \bar{n}_{i}, k_{i}, \rho_{i}\right.$, and so forth).

When the parameter used to described the curve is the hyperquadric arc we shall write $\bar{\Re}=\bar{\Re}(\Im)$ to denote the curve. The notation for the derivatives with respect to $\mathfrak{S}$ and for the hyperquadric tangent, normals, and so forth will remain the same as in the previous section of this paper.

Consider a function $f$ of $\bar{r}(s), \bar{t}(s), \bar{n}_{i}(s), k_{i}(s), d s$, and their derivatives with respect to $s$. If we denote by $\mathfrak{F}$ the same function of $\bar{\Re}(\Im)$, $\overline{\mathfrak{I}}(\mathfrak{S}), \overline{\mathfrak{N}}_{i}(\mathfrak{S}), \boldsymbol{\Re}_{i}(\mathfrak{S}), d \mathfrak{S}$, and their derivatives with respect to $\mathfrak{S}$ and if $f=\mathfrak{F}$, then we say that $f$ is an invariant. 
THEOREM 3.1. For the curve $\bar{r}(s)=\bar{\Re}(S)$ we have the invariant $\phi d s=\Phi d \subseteq$ where $\phi=\left(k_{1}^{n-1} k_{2}^{n-2} \cdots k_{n-2}^{2} k_{n-1}\right)^{2 / n(n+1)}$ and $\Phi$ is the same function of the $\Omega^{\prime} s$.

The proof follows immediately from transformation of parameter in the determinant $\left[\bar{r} \cdot, \bar{r} \cdot \cdot, \cdots, \bar{r}^{(n)}\right]$.

Definition 3.1. The parameter $\sigma$ defined by $d \sigma=\phi d s=\Phi d \subseteq$ will be referred to as the invariant parameter.

THEOREM 3.2. The vectors $\bar{a}_{1}, \cdots, \bar{a}_{n}$ defined by $\bar{a}_{1}=\bar{t} / \phi, \quad \bar{a}_{2}$ $=d \bar{a}_{1} / d \sigma, \cdots, \bar{a}_{n}=d \bar{a}_{n-1} / d \sigma$ are invariant and linearly independent.

They are seen to be invariant from the equation $\bar{a}_{1}=\bar{t} / \phi=\overline{\mathfrak{T}} / \Phi$. To show them linearly independent we first write

$$
\bar{a}_{i}=\sum_{j=0}^{i-1} x_{i j} \bar{n}_{i} \quad\left(\bar{n}_{0}=\bar{t}, i=1, \cdots, n\right)
$$

where the $x_{i j}$ are obtained from the Frenet formulas as functions of $k_{i}(i=1, \cdots, n-1)$. Then if we form the determinant of their components we obtain

$$
\left[\bar{a}_{1}, \cdots, \bar{a}_{n}\right]=\gamma
$$

$$
\text { where from (3.1) } \gamma= \pm 1=\operatorname{sgn}\left[\bar{t}, \tilde{n}_{1}, \cdots, \bar{n}_{n-1}\right]
$$

which shows them to be linearly independent.

Now if we take the derivative of the determinant (3.2) we obtain $\left[\bar{a}_{1}, \bar{a}_{2}, \cdots, d \bar{a}_{n} / d \sigma\right]=0$ and therefore can write $d \bar{a}_{n} / d \sigma=\mu_{1} \bar{a}_{1}+\cdots$ $+\mu_{n-1} \bar{a}_{n-1}$ where the $\mu_{i}$ are invariant. By substitution from (3.2) we obtain

$$
\begin{aligned}
\mu_{1} & =\gamma\left[d \bar{a}_{n} / d \sigma, \bar{a}_{2}, \cdots, \bar{a}_{n}\right], \\
\mu_{2} & =\gamma\left[\bar{a}_{1}, d \bar{a}_{n} / d \sigma, \cdots, \bar{a}_{n}\right], \\
\cdot \cdot \cdot \cdot \cdot \cdot \cdot \cdot \cdot \cdot \cdot, & \cdot \cdot \cdot, \bar{a}_{n-1}=\gamma\left[\bar{a}_{1}, \bar{a}_{2}, \cdots, d \bar{a}_{n} / d \sigma, \bar{a}_{n}\right] .
\end{aligned}
$$

If we use the equations (3.1) we may compute the $\mu_{i}$ in terms of the ordinary curvatures of a curve and since the $\mu_{i}$ are invariant we may write

$$
\mu_{i}\left(\Re_{1}, \cdots, \Re_{n-1}\right)=\mu_{i}\left(\rho_{1}, \cdots, \rho_{n-1}\right) \quad(i=1, \cdots, n-1) .
$$

The functions $\mu_{i}$ may also be shown to define a curve up to initial conditions and hence we may state the following theorem.

THEOREM 3.3. The equations (3.3) may be looked upon as defining the $\Re_{i}$ in terms of the $\rho_{i}$. 
TheOREM 3.4. A necessary and sufficient condition that a curve lis on a real central hyperquadric is that the equation (2.4) is satisfied at each of the points of the curve where the $\mathfrak{G}_{i}$ are given by equations (2.5) and $\Re_{1}, \cdots, \Re_{n-1}$ are to be computed by means of the equations (3.3).

The proof is based on the result of Theorem 2.3 and using equations (3.3) as well as $d \mathfrak{S}=(\phi / \Phi) d \subseteq$ to eliminate the coefficients $a_{b c}$ of the given hyperquadric from equation (2.4).

TheORem 3.5. A necessary and sufficient condition that a curve lie on any hypercone is that at least one of the equations (2.6) is satisfied at each of the points of the curve where the $\mathfrak{S}_{i}$ are given by equations (2.5) and $\Re_{1}, \cdots, \Re_{n-1}$ are to be computed by means of the equations (3.3).

The proof is essentially the same as that of Theorem 3.4.

Sacramento State College 